GT2017-63041

\title{
TIME-RESPONSE OF RECENT PREFILMING AIRBLAST ATOMIZATION MODELS IN AN OSCILLATING AIR FLOW FIELD
}

\author{
G. Chaussonnet; A. Müller; S. Holz, R. Koch, H.-J. Bauer \\ Institut für Thermische Strömungsmaschinen \\ Karlsruher Institut für Technologie (KIT) \\ Kaiserstr. 12 \\ 76131 Karlsruhe, Germany
}

\begin{abstract}
The present study investigates the response of recent primary breakup models in the presence of an oscillating air flow, and compares them to an experiment realized by Müller and coworkers in 2008. The experiment showed that the oscillating flow field has a significant influence on the Sauter Mean Diameter (SMD) up to a given frequency. This observation highlights the low-pass filter character of the prefilming airblast atomization phenomenon, which also introduces a significant phase shift on the dynamics of SMD of the generated spray. The models are tested in their original formulations without any calibration in order to assess their robustness versus different experiments in terms of SMD and time-response to an oscillating flow field. Special emphasis is put to identify the advantages and weaknesses of theses models, in order to facilitate their future implementation in CFD codes. It is observed that some models need an additional calibration of the time constant in order to match the time shift observed in the experiment, whereas some others show a good agreement with the experiment without any modification. Finally, it is demonstrated that the low-pass filter character of the breakup phenomenon can be retrieved by considering the history of the local gas velocity, instead of the instantaneous velocity. This might result in a higher simulation fidelity within CFD codes.
\end{abstract}

\footnotetext{
*geoffroy.chaussonnet@kit.edu

${ }^{\dagger}$ Dr. A. Müller is currently Team leader for sensor systems at JENOPTIK Robot GmbH
}

\section{NOMENCLATURE} Symbols

A Area

C Empiric constant

$D \quad$ Mean diameter

$H$ Height

$U \quad$ Bulk velocity

a Acceleration

$d \quad$ Droplet diameter

$f \quad$ Frequency

$h \quad$ Film thickness

$u \quad$ Local velocity
Greek Symbols

$\Gamma$ Gamma function

$\Lambda$ Volumetric 2D mass flow rate

$\delta \quad$ Characteristic gas thickness

$\lambda$ Wavelength

$\mu \quad$ Dynamic viscosity

$v \quad$ Kinematic viscosity

$\rho$ Density

$\sigma$ Surface tension

$\tau$ Characteristic time

$\varphi$ Phase angle of the siren

$\phi \quad$ Phase shift
Nondimensional numbers

Oh Ohnesorge number

Re Reynolds number

We Weber number

\author{
Abbreviations \\ LDA Laser Doppler Anemometry \\ PDF Probability Density Function \\ VPDF Volume Probability Density Function \\ SMD Sauter Mean Diameter
}




\section{INTRODUCTION}

Lean Premixed Prevaporized combustion is prone to thermoacoustic instabilities [1] which originate from an unsteady interaction between acoustics and heat release. Under certain circumstances, this interaction may lead to a resonance featuring strong fluctuations of the heat release and high amplitude pressure waves. The consequences of thermo-acoustic instabilities span from disturbing effects such as flame blow off and structural vibration, to dramatic effects such as flame flashback and partial mechanical destruction of the combustor. From a hydrodynamic point of view, the pressure fluctuations lead to a strong pulsation of the gas flow, that significantly disturbs the flow pattern inside the combustion chamber and through the fuel injector nozzle.

The operating principle of prefilming airblast atomization is the momentum transfer from a high speed gas flow to the liquid phase of a thin film. This type of injector was previously investigated under fluctuating gas velocity conditions [2-5] and was found to be sensitive to flow fluctuations $[4,5]$. Consequently, typical spray characteristics such as the droplet flux and the Sauter Mean Diameter might also be influenced by thermoacoustic instabilities. Furthermore, since the fuel is delivered by the injector into the combustion chamber, another feedback loop between thermo-acoustic instabilities and atomization process has been observed [6]. Finally, the flow field through the nozzle may also fluctuate due to independent hydrodynamic instabilities such as the Precessing Vortex Core or due to transitions between different flow regimes.

In order to (i) predict the complex interactions between prefilming airblast atomizers and thermo-acoustic instability, and to (ii) capture transient effects in the combustion chamber in numerical simulations, it is necessary to use sophisticated primary breakup models that accurately predict the spray characteristics, both in steady state and in fluctuating flow conditions. The objectives of the present work is to assess recent prefilming airblast atomization models in a pulsated flow field and to study their timeresponse in terms of Sauter Mean Diameter against an experiment realized by Müller and coworkers [4]. Note that no CFD calculations were conducted in the present study, in the atomization models all input variables were set to constant values, except the fluctuating gas velocity which is provided by Müller's experiment.

The experiment will be presented in the first part of the paper, followed by a description of the selected primary atomization models. A comparison of these models with the steady state experiment is made in the third part and their time-response is assessed in the fourth part.

\section{EXPERIMENT}

\section{Test rig and Model Prefilming Airblast Atomizer}

The atomizer considered in this work was investigated by Müller et al. [4]. It consists of a planar prefilmer (Fig. 1) immersed in a high-speed air stream. The liquid is supplied at a volumetric $2 \mathrm{D}$ mass flow rate $\Lambda_{f}$ of $25 \mathrm{~mm}^{2} / \mathrm{s}$ through fifty equidistant holes. This arrangement ensures a uniform lateral wetting of the surface. The liquid builds up a thin film which is driven by the shear force imposed by the gas flow. The liquid film is advected to the trailing edge where it is accumulated. This accumulation eventually will detach from the trailing edge and undergo a breakup $[7,8]$. The investigated liquid is a fuel substitute (Shellsol D70) of surface tension $\sigma=0.025 \mathrm{~N} / \mathrm{m}$, viscosity $\mu_{l}=1.56 \mathrm{mPas}$ and density $\rho_{l}=770 \mathrm{~kg} / \mathrm{m}^{3}$. These values are kept constant in the following.

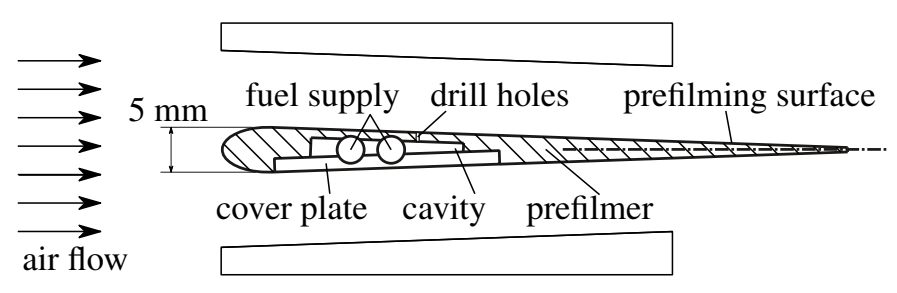

FIGURE 1: Planar model of the airblast atomizer, from [9].

The air flow is supplied at ambient temperature and pressure with a bulk velocity $U_{g}$ of $60 \mathrm{~m} / \mathrm{s}$. The pulsating device, presented in [4], is a siren that generates velocity fluctuations up to an amplitude $u_{g}^{\prime} / U_{g}$ of $50 \%$ and a frequency of $570 \mathrm{~Hz}$. Two fluctuation frequencies $(f=62$ and $500 \mathrm{~Hz})$ are selected and will be discussed in this study. The former frequency corresponds to the lower bound of a resonance frequently observed in combustion chambers [2] and referred to as rumble, while the latter frequency is representative of the first longitudinal mode of usual combustion chambers [5].

\section{Diagnostic technique}

The transient velocity of the gas phase was measured by means of Laser Doppler Anemometry (LDA) $18 \mathrm{~mm}$ downstream the trailing edge and the breakup of the liquid phase was captured by an extended Particle Lagrangian Tracking Velocimetry (PLTV) post-processed by an in-house tool [4]. This tool is based on droplet contour recognition. It allows to account for non-spherical liquid blobs, from which it is possible to derive an equivalent diameter. However, some liquid blobs are so distorted that the equivalent diameter does not have any physical meaning. These liquid blobs were disregarded. Overall, less than $1 \%$ of the liquid blobs were sorted out. 


\section{Experimental results}

Figure 2 shows the measured Volume Probability Density Function (VPDF) of the spray generated downstream the trailing edge for static conditions (i.e. a constant gas velocity of $60 \mathrm{~m} / \mathrm{s}$ ), superimposed with three typical functions used to describe spray size characteristics [10]. The shape of this distribution is well captured by all three functions, with a slight advantage for the Log-Normal function. The $D_{V 10}$, SMD and $D_{V 90}$ are 71, 120 and $280 \mu \mathrm{m}$, respectively.

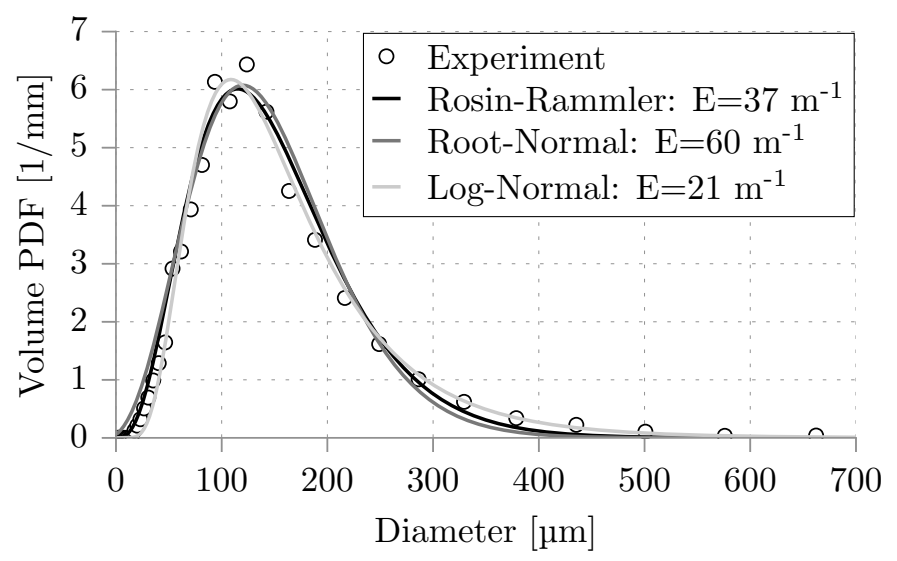

FIGURE 2: Volume PDF of the spray superimposed with usual functions. E is the fitting error defined as $\int\left(f_{\text {exp }}-f_{f i t}\right)^{2} \mathrm{~d} d$.

For transient conditions, the gas velocity is plotted versus the phase of the siren in Fig. 3 (top) for different frequencies. It is observed that the relative amplitude of the velocity oscillation peaks to $112 \%$ at $f=125 \mathrm{~Hz}$ and decreases to $26 \%$ at $f=500 \mathrm{~Hz}$. In a same manner, the phase shift between the gas velocity and the siren increases from $4.2^{\circ}$ to $191^{\circ}$ between 62 and $500 \mathrm{~Hz}$. These characteristics are similar to a second order filter and correspond to the frequency response of the pulsating unit, as shown by Müller [9] with a OD model.

The SMD is depicted in Fig. 3 (bottom) and shows a clear dependency on the phase of the siren, indicating that a fluctuating flow field has a strong impact on the spray generation within this frequency range. Furthermore, the SMD presents the same frequency features as the gas velocity, i.e. a decreasing amplitude and an increasing phase shift at higher frequencies. However, it is assumed that these trends originate from both the variations of the gas velocity, which drives the breakup process, and the frequency response of the liquid reservoir at the tip of the trailing edge itself. Therefore the amplitude and the phase shift of the SMD variations are normalized by the gas velocity variation, in terms of a transfer function $F(t)=S M D(t) / u_{g}(t)$. The gain $\mathrm{G}$ is based on the ratio of each relative amplitude $A_{r}$ such as $G=A_{r}(S M D) / A_{r}\left(U_{g}\right)$ and the phase shift $\phi$ is computed from the arccosine of the cross correlation of the two signals. It is found that $G$ reaches a maximum of 0.98 at $f=125 \mathrm{~Hz}$ and decreases to 0.032 at $f=500 \mathrm{~Hz}$ while $\phi$ decreases monotonously from $160^{\circ}$ at $f=62 \mathrm{~Hz}$ to $103^{\circ}$ at $f=500 \mathrm{~Hz}$. The evolution of the gain is similar to a second order showing a resonance at $\approx 125 \mathrm{~Hz}$.

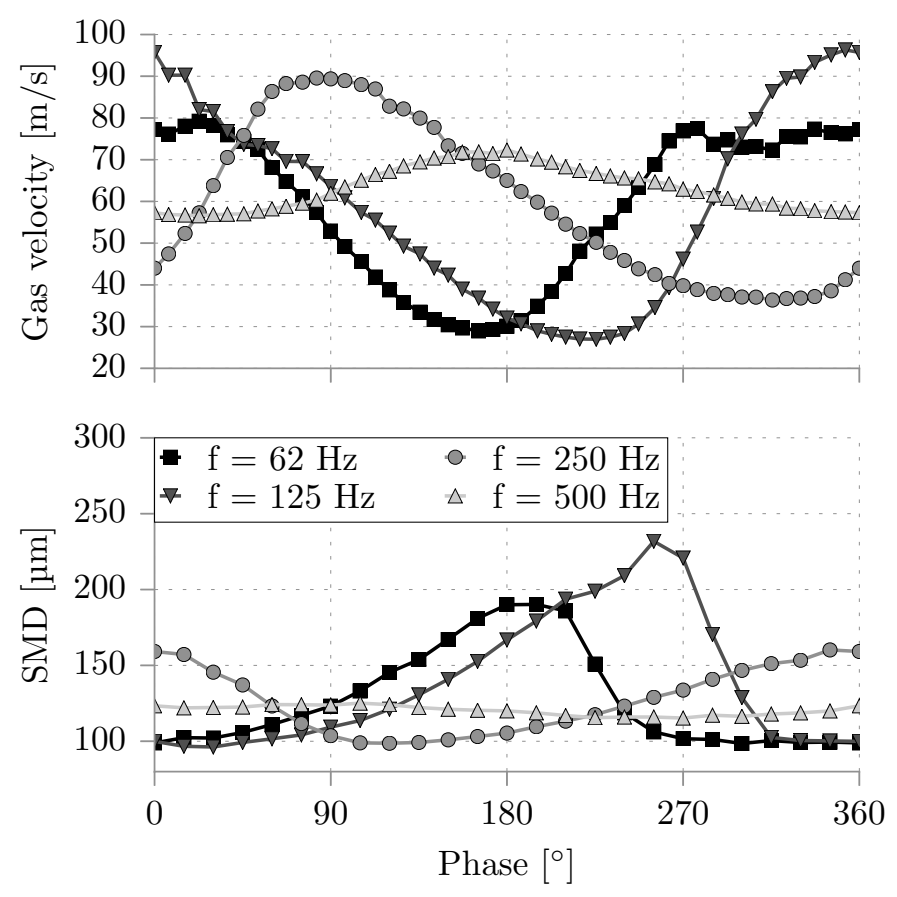

FIGURE 3: Gas velocity (top) and SMD (bottom) of the generated spray.

The SMD is plotted versus the gas velocity for different frequencies in Fig. 4, and highlights the unsteady effects of the spray generation. Each closed curve corresponds to a operating point excited at a constant frequency. First, it is observed that each operating point is represented by a distinct loop. None is reduced to a single line. This means that one gas velocity can lead to different SMDs, and highlights the hysteresis of this type of breakup in transient conditions. Furthermore, the lower bound of all operating points collapses to the same line represented by the equation $y=A x^{B}$. This means that, at the lower bound, the SMD is only dependent on the gas velocity and not on the excitation frequency. This domain corresponds to a breakup similar to a breakup in steady state condition. The data points which are located off this domain characterize the unsteady part of the breakup. The amount of data points located in the unsteady zone increases with increasing frequency. Indeed, the curve of $f=500 \mathrm{~Hz}$ is almost out of the steady state domain, suggesting that the fluctuations time scale is significantly lower than the 
breakup time. Finally, the area delimited by each loop illustrates the loss of atomization efficiency due to the phenomenon of unsteady breakup. Indeed, in the unsteady zone, the SMD is always larger than in the steady state zone, leading to a coarser spray compared to a spray generated in steady state conditions.

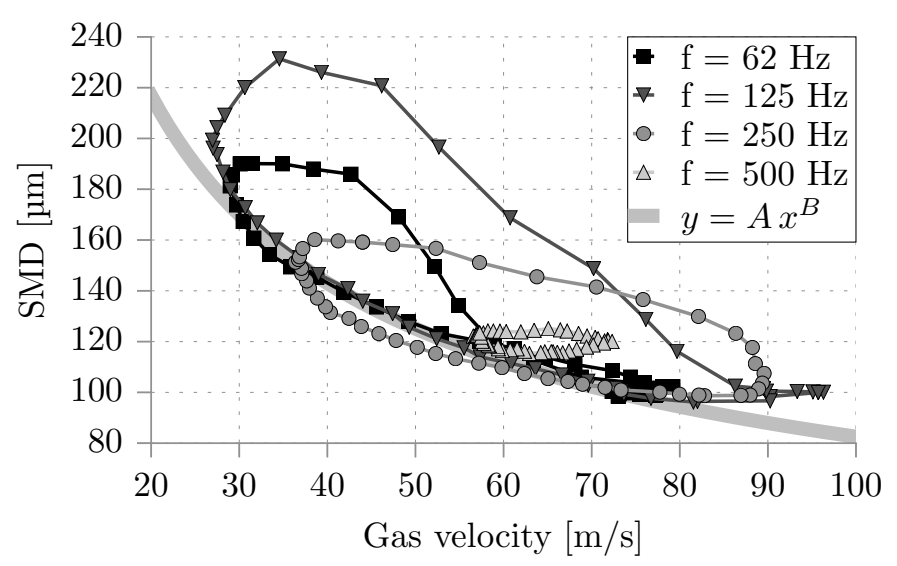

FIGURE 4: SMD of the generated spray versus the gas velocity, superimposed with the line of equation $y=1308 x^{-0.6}$.

\section{RECENT MODELS FOR PREFILMING AIRBLAST AT- OMIZATION}

Several advanced primary breakup models for prefilming airblast atomization were developed in the last five years. Three of the most relevant ones are presented in the following. In comparison to older approaches that provide only a characteristic droplet diameter, these models predict the whole volume and/or number PDF, which represents a step further in the prediction of prefilming airblast atomization.

\section{Model 1}

Model 1 was developed by Inamura et al. [11] and calibrated using their own experiment in which water was atomized under various conditions of liquid mass flow rate and gas velocity. The droplet size distribution was measured $50 \mathrm{~mm}$ downstream the trailing edge by means of laser diffraction. The proposed mechanism for prefilming airblast atomization is depicted on Fig. 5. Longitudinal waves of length $\lambda_{1}$ develop on the film surface according to the Kelvin-Helmholtz instability, and are longitudinally accelerated by the gas, triggering a transverse RayleighTaylor instability of wavelength $\lambda_{2}$. When these structures reach the trailing edge, they disintegrate by a bag breakup mechanism. Inamura et al. [11] neglected the fine droplets generated by the membrane and focused on the rim of the bag, which was decomposed into three cylinders of diameter $d_{1}$ forming a ' $U$ '-shape, and fragmenting themselves into blobs of diameter $d_{d}$ following Weber's theory. In turn, these large blobs will then be disintegrated according to the ligament breakup described by Marmottant and Villermaux [12], forming a spray whose drop size number distribution $f_{0}$ follows a Gamma function. Inamura et al. [11] used additionally the Taylor Analogy Breakup (TAB) model [13] to take secondary atomization into account.

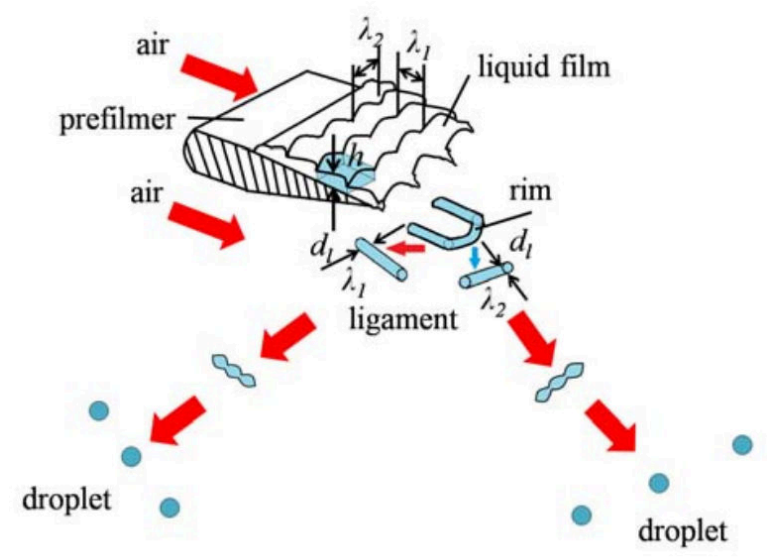

FIGURE 5: Breakup mechanism proposed by Inamura et al. [11].

The above-mentioned quantities are computed by:

$$
\begin{array}{r}
\lambda_{1}=C_{1} \delta \sqrt{\frac{\rho_{l}}{\rho_{g}}} \text { with } \delta=3.56 \frac{H_{g}}{\sqrt{\mathrm{Re}_{H_{g}}}} \\
\lambda_{2}=2 \pi \delta \sqrt{\frac{6 C_{1} C_{2}}{C_{d}}}\left(\frac{\rho_{l}}{\rho_{g}}\right)^{1 / 4} \sqrt{\frac{\sigma}{\rho_{g}\left(U_{g}-U_{l}\right)^{2} \delta}} \\
d_{1}=2 \sqrt{\frac{\lambda_{1} \lambda_{2} h}{\pi\left(2 \lambda_{1}+\pi \lambda_{2} / 2\right)}} \text { and } d_{d}=1.88 d_{1}(1+3 \mathrm{Oh})^{1 / 6} \\
f_{0}(x)=\frac{n^{n}}{\Gamma(n)} x^{n-1} e^{-n x} \quad \text { with } \quad x=\frac{d}{d_{d}}
\end{array}
$$

where $\delta$ is the boundary layer thickness above the film at the trailing edge, $h$ is the film thickness and $C_{d}$ the drag coefficient of the waves. The symbols $\mathrm{Re}_{H_{g}}$ and Oh represent the Reynolds number $U_{g} H_{g} / v_{g}$ based on the gas channel height and the Ohnesorge number $\mu_{l} / \sqrt{\rho_{l} \sigma d_{l}}$, respectively. As illustrated later, the TAB model is active for all droplets and, therefore, leads to a global down-scaling of the drop size distribution. More details on the TAB model are given in [13].

Model 1 uses four constants $C_{1}, C_{2}, C_{d}$ and $n$ that were originally 
set to $1.6,0.25,2.01$ and 4 , respectively. Note that $C_{1}$ was determined experimentally by Boukra et al. [14] and $n$ was found to vary between 2.5 and 3.5 [12]. In addition, since $C_{d}$ only appears together with $C_{2}$ (Eq. 1b), the dependance on $C_{d}$ is incorporated into $C_{2}$ in the following.

The model in its original formulation does not contain any time scale estimation of the primary breakup. This is introduced in the present work. It is assumed that the breakup time is the sum of the cylinder and the ligament breakup time. The former is evaluated with a Linear Stability Analysis by Weber [15] to $\tau_{c y l} \approx-2.545 \log \left(\eta_{0}\right) \sqrt{\rho_{l} d_{1}^{3} / 8 \sigma}$ while the latter is measured [12] to $\tau_{\text {liga }} \approx \sqrt{\rho_{l} d_{d}^{3} / \sigma}$. The term $\eta_{0}$ corresponds to the relative initial perturbation of the cylinder radius and is set here to 0.1 . Note that the time scale of the secondary breakup given by the TAB model is below $10 \mu$ s, which is negligible. Finally :

$$
\tau_{\text {tot }}=\tau_{c y l}+\tau_{\text {liga }}=\sqrt{\frac{\rho_{l}}{\sigma}}\left(\sqrt{d_{d}^{3}}-2.545 \log \left(\eta_{0}\right) \sqrt{\frac{d_{1}^{3}}{8}}\right)
$$

The set of Eqs. 1 depends on the geometric features of the injector, on the physical properties of fluid, and on the film thickness. These quantities are accessible within any CFD codes. However, the bulk gas velocity $U_{g}$ should be replaced by a local velocity in order to take into account local transient flow states predicted by numerical simulations. Such modification is beyond the scope of the present study.

\section{Model 2}

Model 2 was derived by Eckel et al. [16] and calibrated by the experiment of Gepperth et al. [7,8], similar to the present study. In this experiment, prefilming airblast atomization was studied directly downstream the trailing edge by means of shadowgraphy and image processing. The gas velocity, liquid properties as well as the trailing edge thickness were investigated. The proposed mechanism, illustrated in Fig. 6 is explained as follows. The film is driven by the shear forces imposed by the gas. Longitudinal waves of wavelength $\lambda_{\text {stream }}$ appear on the film surface and are convected to the trailing edge, where the liquid is accumulated. A spanwise undulation of wavelength $\lambda_{\text {span }}$ is created mainly by capillarity forces. Both longitudinal and spanwise waves define a volume of liquid. It is assumed that a droplet of a corresponding equivalent diameter will be atomized in a bag breakup mode. Contrary to Model 1, Model 2 takes also the fine droplets generated by the membrane fragmentation into account, resulting in a bimodal PDF. Hence, it is assumed that the bag and the rim (or ligament) breakup lead to independent drop size VPDFs, both described by a Root-Normal (RN) distribution but with different scales and widths, $f_{3, b a g}$ and $f_{3, \text { liga }}$, respectively. The expression of the $\mathrm{RN}$ distribution yields:

$$
f_{3}(m, q, d)=\frac{A}{\sqrt{d}} \exp \left[-\frac{1}{2}\left(\frac{\sqrt{d}-\sqrt{m}}{\sqrt{q}}\right)^{2}\right]
$$

where $A, m$ and $q$ are a normalizing factor, the scale and the width of the distribution, respectively. The global spray VPDF $f_{3, T}$ is the superposition of $f_{3, \text { bag }}$ and $f_{3, \text { liga }}$. In order to parametrize these distributions, Eckel et al. [16] use a Sauter Mean Diameter $(S M D)$ and Mean Mass Diameter (MMD, also referred to as $D_{V 50}$ in the literature), based on the work of Tate and Marshall [17], Faeth and coworkers [18, 19], and Wert [20].

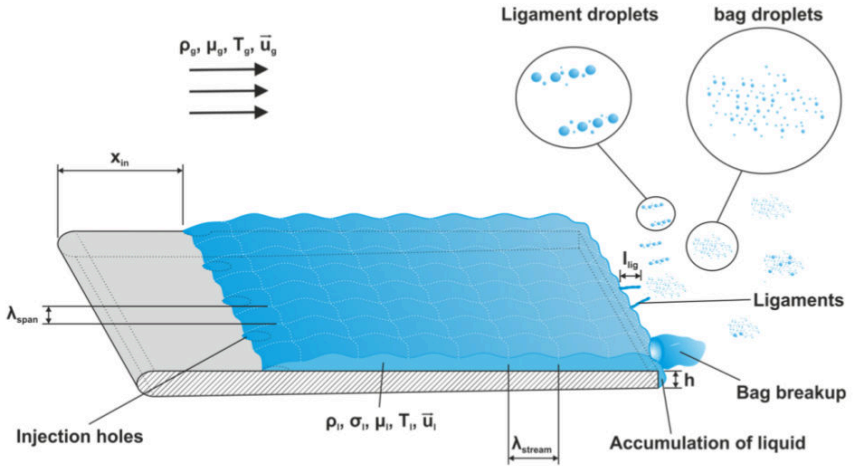

FIGURE 6: Breakup mechanism proposed by Eckel et al. [16].

The equations of the model are:

$$
\begin{array}{r}
\lambda_{\text {stream }}=\delta_{\omega} \sqrt{\frac{\rho_{l}}{\rho_{g}}} \text { with } \delta_{\omega}=8.5 \frac{H_{g}}{\sqrt{\operatorname{Re}_{L_{p}}}} \\
\lambda_{\text {span }}=2 \sqrt{\frac{\Lambda_{f} \lambda_{\text {stream }}}{\pi U_{c}}} \text { where } U_{c}=U_{g} \sqrt{\frac{\rho_{g}}{\rho_{l}}} \\
d_{0}=\sqrt[3]{6 V_{0} / \pi} \quad \text { where } \quad V_{0}=\Lambda_{f} \lambda_{\text {stream }} \lambda_{\text {span }} / U_{c} \\
S M D_{\text {bag }}=0.044 d_{0} \quad \text { and } M M D_{\text {bag }}=1.04 S M D_{\text {bag }} \\
S M D_{\text {liga }}=0.32 \frac{\sigma}{\rho_{g} U_{r}^{2}}\left[\mathrm{We}_{d_{0}}\left(t_{\text {ini }}^{*}-t_{\text {liga }}^{*}\right)\right]^{2 / 3} \\
M M D_{\text {liga }}=C_{1} S M D_{\text {liga }} \\
f_{3, T}(d)=C_{2} f_{3, \text { bag }}(d)+\left(1-C_{2}\right) f_{3, \text { liga }}(d)
\end{array}
$$

The term $\delta_{\omega}$ represents the vorticity thickness of the gas flow at the trailing edge and is expressed by a Reynolds number based on the prefilmer length $L_{p}$ as $\operatorname{Re}_{L_{p}}=U_{g} L_{p} / v_{g}$. The film volumetric $2 \mathrm{D}$ flow rate is referred to as $\Lambda_{f}, U_{c}$ is the velocity of the 
longitudinal waves and $U_{r}$ is the gas/liquid relative velocity. In Eq. $4 \mathrm{e}$, the Weber number yields $\mathrm{We}_{d_{0}}=\rho_{g} U_{g}^{2} d_{0} / \sigma$, and $t_{i n i}^{*}$ and $t_{\text {base }}^{*}$ stand for the nondimensional initiation and breakup times, respectively. They are given by:

$$
\begin{aligned}
t_{\text {ini }}^{*} & =t_{\text {ini }} / t^{*}=1.6 / \chi \text { and } t_{\text {liga }}^{*}=t_{\text {liga }} / t^{*}=C_{3} / \chi \\
t^{*} & =\frac{d_{0}}{U_{r}} \sqrt{\frac{\rho_{l}}{\rho_{g}}} \\
\chi & =1 \text { for } \text { Oh }<0.1 \\
\chi & =1-\text { Oh } / 7 \text { for } \text { Oh }>0.1
\end{aligned}
$$

where Oh is the Ohnesorge number based on $d_{0}$ and $C_{3}$ is a constant equal to 5 in $[18,19]$ that will be varied in the present study. Since the liquid wave pattern of dimension $\lambda_{\text {stream }} \times \lambda_{\text {span }}$ is generated on the film, only the time scale of the bag breakup is considered by the model.

The model is based on three arbitrary constants $C_{1}$ to $C_{3}$ but also on several empirical constants measured in various experiments. Like for Model 1, it takes as input geometrical features of the atomizer, the physical properties of the fluid and the bulk gas velocity. It is therefore subject to the same weakness in case of gas velocity local fluctuations and might need a local formulation. Since the Cumulative Distribution Function (CDF) of the RootNormal distribution $F_{3}(d)$ is the Error Function erf(), it can be inverted so that an equiprobable random draw $X$ between 0 and 1 allows to generate random numbers following the Root-Normal distribution in a straightforward manner:

$$
d=F_{3}^{-1}(X) \quad \text { with } \quad F_{3}\left(F_{3}^{-1}(X)\right)=X
$$

However, in the approach of Eckel et al., the SMD and the MMD are used to characterize the distribution. The parameter $m$ is straightforward with $m=$ MMD but the SMD does not explicitly appear in Eq. 3 which means that $q$ must be fitted in order to retrieve the SMD. In the context of embedding this model into a CFD code, it means that the fitting must be applied for each injected droplet, which is computationally demanding and turns out to be the weakest point of this model. However, in the range of parameters investigated here, the ratio $m / q$ was found to be strictly constant to $1.2510^{-2}$ and roughly constant to $5.7810^{-2} \pm$ $2.56 \%$ for the bag and ligament breakup respectively. Hence for a pragmatic use of this model, $m / q$ would be to set as constant.

\section{Model 3}

Model 3, named PAMELA which stands for Primary Atomization Model for prEfiling airbLAst injectors, was developed by Chaussonnet et al. [21,22] and calibrated by the experiments of Gepperth et al. [7, 8] described previously. The proposed mechanism is depicted in Fig. 7. The film flow feeds the liquid reservoir (i) which is partly immersed into the high-speed gas flow (ii). The surface of the liquid reservoir is sheared by the highspeed air stream and is strongly accelerated (iii) in the longitudinal direction, leading to a spanwise Rayleigh-Taylor instability of wavelength $\lambda_{R T}$ (iv). These spanwise undulations are blown up by the gas (v), and disrupt into bags and ligaments (vi).

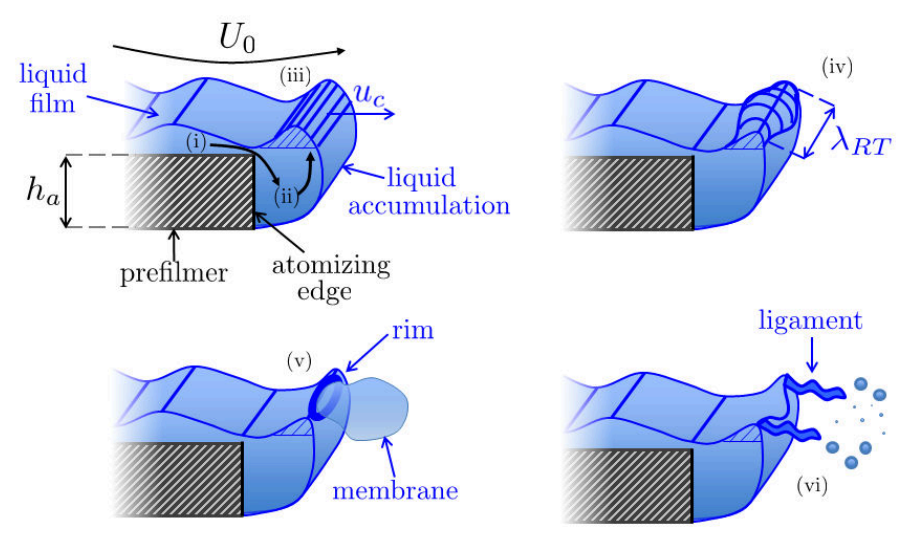

FIGURE 7: Breakup mechanism proposed by Chaussonnet et al. [22].

Contrary to Model 1 and 2, in this approach it is assumed that there is a decoupling between the film flow and the breakup process, as it was observed in the experiment. The waves formed on the film surface do not have an effect and, hence, are not considered. It is also assumed that the SMD of the spray is proportional to $\lambda_{R T}$ and the drop size PDF can be described by a Rosin-Rammler (RR) distribution

$$
f_{0}(d)=q m^{-q} d^{q-1} \exp \left[-\left(\frac{d}{m}\right)^{q}\right]
$$

where $m$ and $q$ are the scale and shape parameters, respectively. The model is described by the following equations

$$
\begin{array}{r}
\lambda_{R T}^{h_{a}}=\frac{2 \pi}{r_{\rho} u_{g}^{*}} \sqrt{\frac{6 C_{1} h_{a} \sigma}{\rho_{g}}} \text { with } r_{\rho}=\frac{\sqrt{\rho_{l}}}{\sqrt{\rho_{l}}+\sqrt{\rho_{g}}} \\
m=C_{2} \lambda_{R T}^{h_{a}} \frac{\Gamma(2 / q+1)}{\Gamma(3 / q+1)} \\
q=\frac{C_{3}}{\sqrt{\mathrm{We}_{\delta}}}+\left(\frac{h_{a}}{C_{4}}\right)^{2}+C_{5} \text { with } \mathrm{We}_{\delta}=\rho_{g} \delta u_{g}^{2} / \sigma \\
\tau_{c}=\left(\frac{\sigma}{a^{3}\left|\rho_{l}-\rho_{g}\right|}\right)^{1 / 4} \text { with } a=\frac{1}{2} \frac{1}{C_{1} h_{a}} \frac{\rho_{g}}{\rho_{l}}\left(r_{\rho} u_{g}\right)^{2}
\end{array}
$$

where $h_{a}$ is the trailing edge thickness. The term $u_{g}^{*}$ is a timeaverage of the gas velocity at the breakup location and $\mathrm{We}_{\delta}$ is the 
aerodynamic Weber number based on the boundary layer thickness at the trailing edge $\delta$. The SMD is directly expressed by $\mathrm{SMD}=C_{2} \lambda_{R T}^{h_{a}}$. The capillarity time scale $\tau_{c}$ (Eq. $8 \mathrm{~d}$ ) is expressed in terms of the acceleration $a$ of the liquid crests appearing in the liquid accumulation. The whole time scale of the breakup process is the sum of the Rayleigh-Taylor instability time scale $\tau_{R T}$ and the breakup time scale $\tau_{b u}$. Both time scales are found to be proportional to the capillarity time as $\tau_{R T} \approx 10 \tau_{c}$ and $\tau_{b u} \approx 1.8 \tau_{c}[22]$ so that:

$$
\tau_{\text {tot }}=C_{\tau} \tau_{c} \quad \text { with } \quad C_{\tau} \approx 11.8
$$

Constants $C_{1}$ to $C_{5}$ in Eqs. 8 are essentially independent of the liquid properties or the geometry, and although they were calibrated for a planar geometry, they showed a good agreement when predicting an annular nozzle [23]. Their values are 0.67, $0.112,6.82,5.99[\mathrm{~m}], 0.0177$, respectively.

The inputs of the model are the geometric features of the injector as well as fluid properties. Concerning the dependency on the gas velocity, the model exists in a global and in a local form [22]. The global form takes the bulk velocity $U_{g}$ as an input and estimates $u_{g}$ and $\delta$ by:

$$
u_{g}^{*}=0.7 U_{g} \quad \text { and } \quad \delta=0.16 L_{p} \operatorname{Re}_{L_{p}}^{-1 / 7}
$$

The local mode, formulated to be embedded into CFD codes, uses the law-of-the-wall on the prefilmer surface at the trailing edge to estimate $u_{g}^{*}$ and $\delta$. Further details can be found in [22]. In the present study, the global mode is used. As the model does not rely on any film quantities, it can be implemented without any modeling of the film flow provided that the liquid mass flow rate is given. The quantile (the reciprocal of the CDF) of the RR distribution can be explicitly formulated. Hence a RR random draw is straightforward: given an uniformly distributed random variable $X$ between 0 and 1, the PDF of the random variable defined by $d=m \sqrt[q]{-\log (1-X)}$ follows the law given by Eq. 7 .

A particular aspect of this model is that it is based on phenomenological observation and was developed to retrieve the proper droplet size PDF. It was not intended to describe the liquid accumulation dynamics or the breakup mechanism such as a bag breakup or cylinder breakup.

\section{MODEL COMPARISON IN STEADY STATE}

The models are first tested in a steady state to assess their robustness. Note that Model 2 and Model 3 were calibrated in a configuration very similar to the one presented here. Hence, it is expected that they will show a better agreement. Static conditions are defined as a constant velocity $U_{g}=60 \mathrm{~m} / \mathrm{s}$, $\Lambda_{f}=25 \mathrm{~mm}^{2} / \mathrm{s}$, a film thickness $h_{f}$ of $20 \mu \mathrm{m}$, a channel height
$H_{g}$ and length $L_{p}$ of 8 and $7 \mathrm{~mm}$, respectively, and trailing edge thickness of $0.5 \mathrm{~mm}$.

\section{With original constants}

Figure 8 shows the VPDF as predicted by the three models with their original calibration constants, superimposed with vertical lines that locate the resulting SMD, and with experimental VPDF measured in the static conditions defined earlier.

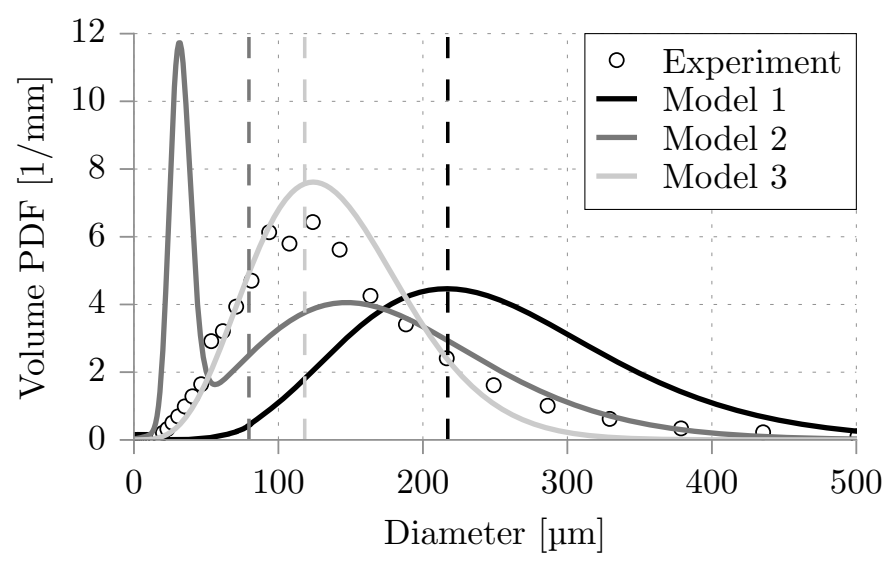

FIGURE 8: Volume PDF with original model constants superimposed with the experiment under static conditions. Vertical dashed lines denote the SMDs.

The SMDs scatter between 80 and $221 \mu \mathrm{m}$. According to its mechanism, Model 2 shows a bimodal distribution with a highly pronounced peak corresponding to the bag breakup, which is explained by the constant $C_{2}=0.2$ leading to $20 \%$ of the spray volume being generated by bag breakup. In Model 1, the time scale of the secondary breakup predicted by the TAB model is so small that all droplets can be considered to undergo secondary breakup instantaneously, as mentioned by Inamura et al. [11]. Note that the use of the TAB model leads to a noisy PDF so that a smoothing convolution with a Gaussian function is applied. To illustrate the impact of the TAB model in Model 1, Fig. 9 shows the VPDF of Model 1 (i) without TAB, (ii) with TAB without smoothing convolution and (iii) with TAB and smoothing convolution, in static conditions. The dashed line corresponds to the line of Model 1 in Fig. 8. The use of the TAB model has a strong influence on the VPDF. The SMD decreases from 504 to $217 \mu \mathrm{m}$ while the convolution leaves the SMD at $217 \mu \mathrm{m}$. According to Fig. 9, it is mandatory to use the TAB model in Model 1 to predict a correct VPDF. 


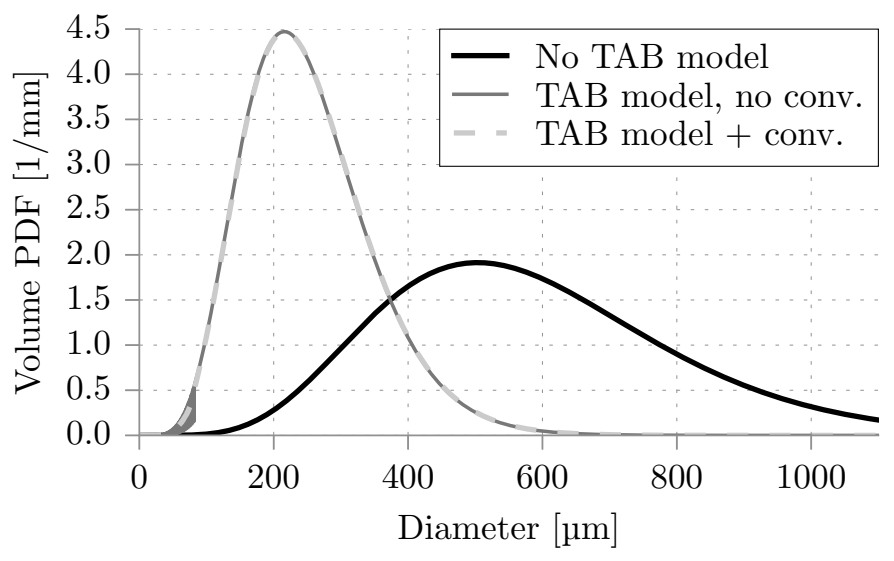

FIGURE 9: Volume PDF of Model 1.

\section{MODEL CALIBRATION TO EXPERIMENTAL DATA}

The constants of the models were fitted on the experimental VPDF using a least squares method, leading to $C_{1}, C_{2}$ and $\mathrm{n}=0.260,8.7710^{-5}$ and 0.112 for Model $1, C_{1}$ and $C_{2}=1.23$ and 0 for Model 2 and $C_{1}$ to $C_{5}=0.651,0.110,5.69,2.3110^{6}$ and -0.236 for Model 3. The output is depicted in Fig. 10. Due to a larger level of complexity induced by the use of the TAB model, the calibration of Model 1 did not satisfactorily fit the experimental curve. This low convergence is also reflected by the large variation of the constants $C_{1}$ and $C_{2}$ compared to their original values, from 1.6 to 0.260 and from 0.25 to $8.7710^{-5}$ respectively. In Model 2, the calibration procedure results in $C_{2}=0$, which is equivalent to neglecting the droplets generated by the membrane of the bag and leads to a unimodal distribution. However the VPDF built from the experiment was averaged into several classes and a secondary peak might have been smoothed by the averaging procedure, and therefore neglecting the secondary peak in Model 2 does not necessarily reflect the entire behavior of the model. For all these reasons, the temporal responses of the models will be studied in the following without calibration.

\section{MODEL COMPARISON IN OSCILLATING FLOW}

The oscillating gas velocity $U_{g}(t)$ was given as input to the models which produce a VPDF depending on $t$. The SMD computed from the VPDF is then given as a function depending on the gas velocity $\operatorname{SMD}\left(U_{g}(t)\right)$. In order to take the delay induced by the breakup into account, the time $\tau$ at which the droplets are effectively generated is defined by adding the breakup time $\tau_{B U}$ calculated at $t$, so that $\tau(t)=t+\tau_{B U}\left[U_{g}(t)\right]$. As larger velocity leads to a lower $\tau_{B U}$, this definition might lead in the case of an increasing velocity to a $\tau(t)$ that decreases with time, as illustrated later.

Figure 11 shows the breakup time as predicted by the models versus the phase $\varphi$, superimposed with the experimental gas

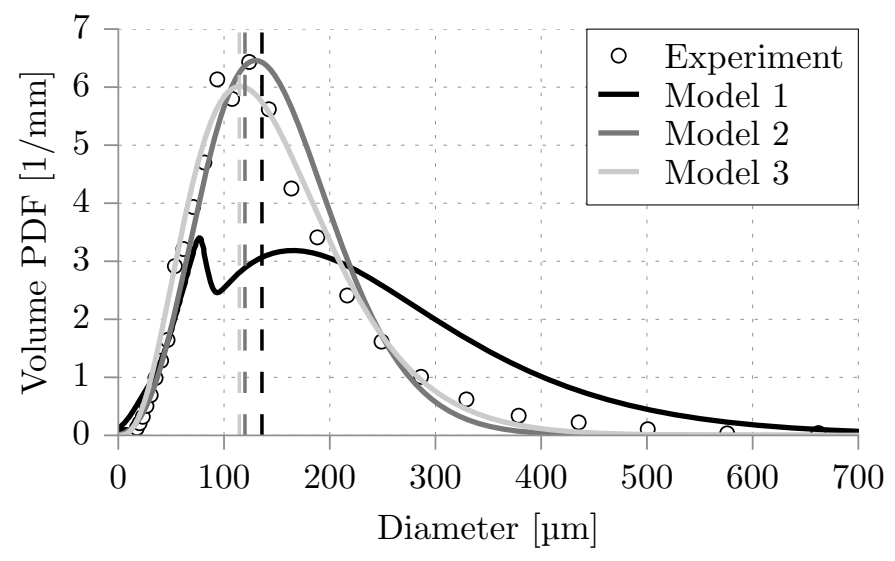

FIGURE 10: Volume PDF with model constants fitted on experiment. Vertical dashed lines denote the SMD.

velocity, for $f=62 \mathrm{~Hz}$ (top) and $500 \mathrm{~Hz}$ (bottom). As the siren rotates at a constant frequency, its instantaneous phase angle can be related to $t$ by $\varphi(t)=2 \pi f t[2 \pi]$. However, the phase angle is here estimated at the effective time of breakup, so that $\varphi(\tau)=2 \pi f \tau(t)[2 \pi]$. The three models show a decreasing breakup time for an increasing velocity. Although they are based on different mechanisms, Model 2 and 3 predict a fluctuation of the breakup time of similar mean value and similar amplitude. Model 1 leads to a lower mean value and a significantly lower amplitude.

Figure 12 shows the SMD as predicted by the models and the experimental data versus the phase $\varphi$ deduced from $\tau(t)$, for $f=62 \mathrm{~Hz}$ (top) and $500 \mathrm{~Hz}$ (bottom). As mentioned earlier, as $\tau(t)$ does not evolve monotonously with $t$, the curve of the SMD versus $\varphi$ (i.e. $\tau(t)$ ) exhibits a hysteresis in time. This is actually an artifact due to the assumed delay between the influence of the gas velocity and the liquid fragmentation. A summing and averaging procedure was tested to consider only one VPDF per time interval, but for particular operating points it led to spurious unphysical SMD oscillations. At the low frequency (Fig. 12 top), the breakup time provided by Model $1(\approx 1 \mathrm{~ms})$ is much lower than the period of the oscillation $(\approx 16 \mathrm{~ms})$ so that the delay is not visible, whereas the two other models predict $\tau_{B U} \approx 6 \mathrm{~ms}$ so that their delay strongly influence the SMD curve. Nevertheless all models show a SMD evolution approximately in phase with the experiment. At high frequency (Fig. 12 bottom), all models present an acceptable SMD amplitude, but locations of maxima and minima are not in phase with the experiment. This means that using the instantaneous velocity as an input for the investigated models leads to large discrepancies. In particular, the low-pass filter characteristic of the breakup phenomenon as highlighted by the experiment is completely neglected by all models. 

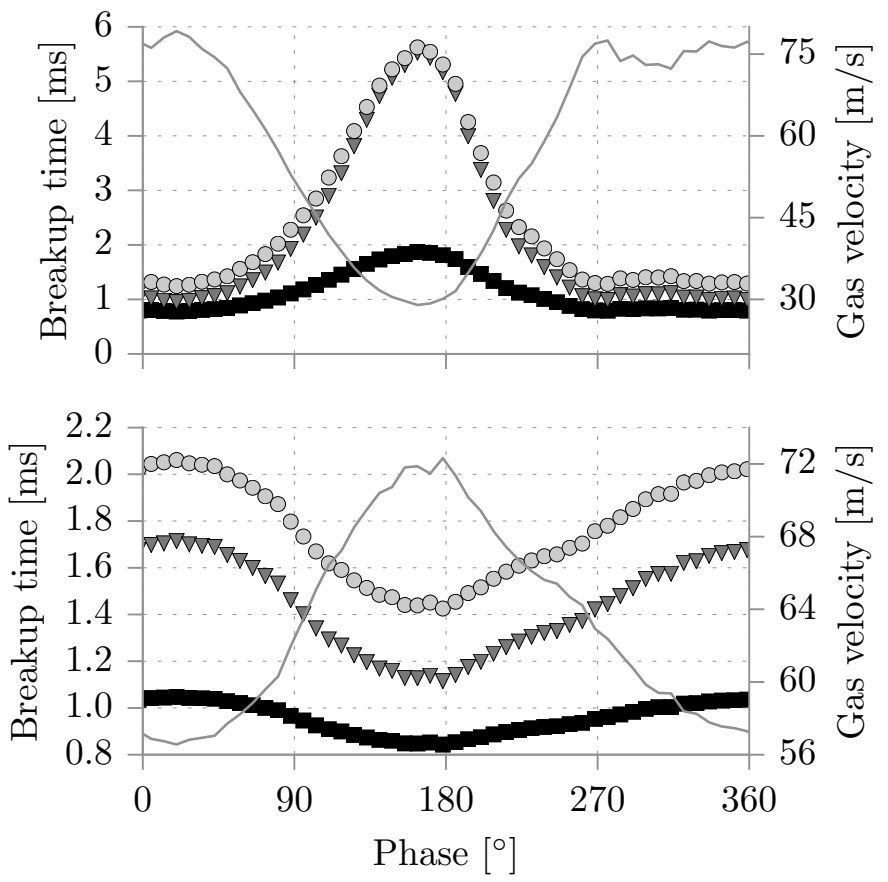

FIGURE 11: Breakup time predicted by Model 1 ( $\square)$, Model 2 $(\nabla)$ and Model $3(\bigcirc)$ superimposed with the gas velocity (line), at $f=62 \mathrm{~Hz}$ (top) and $f=500 \mathrm{~Hz}$ (bottom).

\section{TAKING VELOCITY HISTORY INTO ACCOUNT}

In order to render the low-pass filter effect of the primary breakup phenomenon, the gaseous velocity signal is integrated in time as proposed in Chaussonnet et al. [22]. As the onset of the liquid instability leading to the primary breakup is not instantaneous, it does not depend solely on the instantaneous gas velocity at a given time, but on the whole history of the local gas velocity between the birth and the death of this instability. When the time scale of the gas fluctuations is much larger than $\tau_{B U}$ as in the case of $f=62 \mathrm{~Hz}$, the SMD follows the oscillations of the gas. On the contrary, when the gas fluctuation time scale is comparable to or lower than $\tau_{B U}$, the liquid instability can not follow the rapid changes of the gas, which leads to a SMD time evolution that smoothes out the fluctuations of the gas flow. This phenomenon can be looked at as an averaging process.

The procedure to mimic this effect is sketched in Fig. 13 and explained as follows. At $t_{0}$, the instantaneous gas velocity is used to compute $\tau_{B U}\left[U_{g}\left(t_{0}\right)\right]$. In the following time steps, the mean gas velocity $\bar{U}_{g}^{t_{0}}(t)$ is averaged between $t_{0}$ and the current time $t$, so that it can be seen as a time average with an increasing averaging window. The updated breakup time is computed every time step from $\bar{U}_{g}^{t_{0}}(t)$. At $t=t_{1}$, the averaging time $t_{1}-t_{0}$ is larger than the breakup time $\tau_{B U}\left[\bar{U}_{g}^{t_{0}}\left(t_{1}\right)\right]$ (not depicted in Fig. 13). Hence, the procedure has converged, and the breakup has effectively oc-
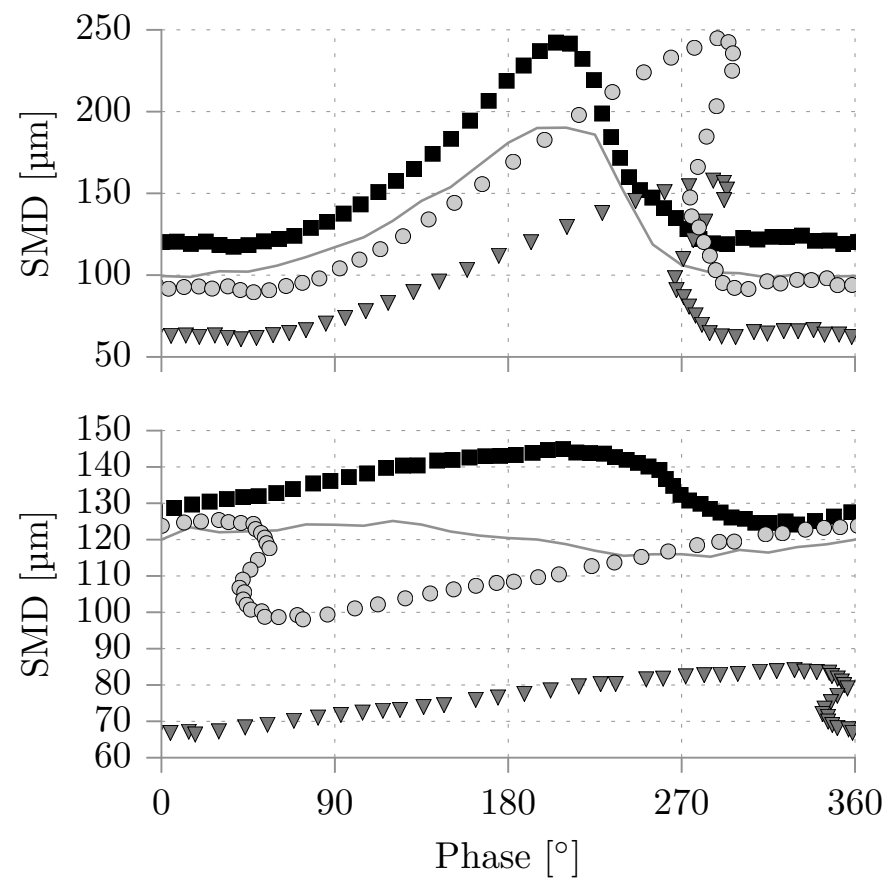

FIGURE 12: SMD predicted by Model $1(\boldsymbol{\square})$, Model $2(\nabla)$ and Model $3(\bigcirc)$ and measured in the experiment (line) at $f=62 \mathrm{~Hz}$ (top) and $f=500 \mathrm{~Hz}$ (bottom).

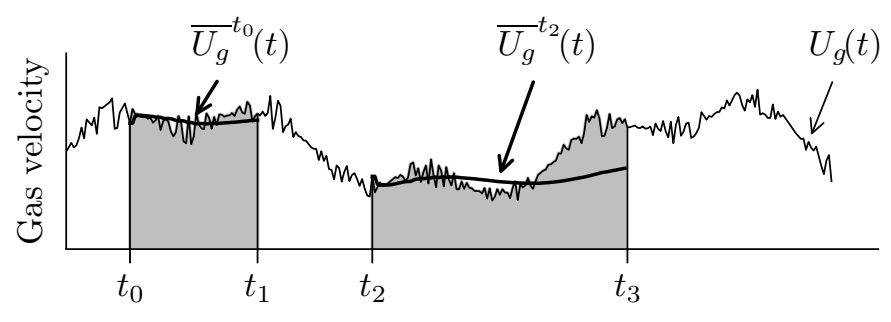

Time

FIGURE 13: Illustration of the averaging procedure for two breakup event.

curred. In a CFD code, this time step would correspond to the creation of a droplet to be injected into the numerical domain. The time of breakup is then defined by $\tau\left(t_{1}\right)=t_{0}+\tau_{B U}\left[\bar{U}_{g}^{t_{0}}\left(t_{1}\right)\right]$ and the corresponding SMD is equal to $\operatorname{SMD}\left[\bar{U}_{g}^{t_{0}}\left(t_{1}\right)\right]$. In Fig. 13, the first breakup event between $t_{0}$ and $t_{1}$ occurs at a larger gas velocity. Hence, it is shorter than the event between $t_{2}$ and $t_{3}$. The breakup time $\tau_{B U}$ of Models 1, 2 and 3 is defined by $\tau_{\text {tot }}$ (Eq. 2), $t_{\text {liga }}$ (Eq. 5a), and $\tau_{\text {tot }}$ (Eq. 9), respectively.

The resulting breakup time is depicted in Fig. 14 for $f=62 \mathrm{~Hz}$ (top) and $500 \mathrm{~Hz}$ (bottom). As expected the averaging procedure has a moderate influence at low frequency while exhibiting 
a strong impact at $f=500 \mathrm{~Hz}$. For Model 2 and Model 3 at low frequency, the curves of $\tau(t)$ show a negative phase shift (phase advance) compared to the gas velocity, which is typical for integral filters.
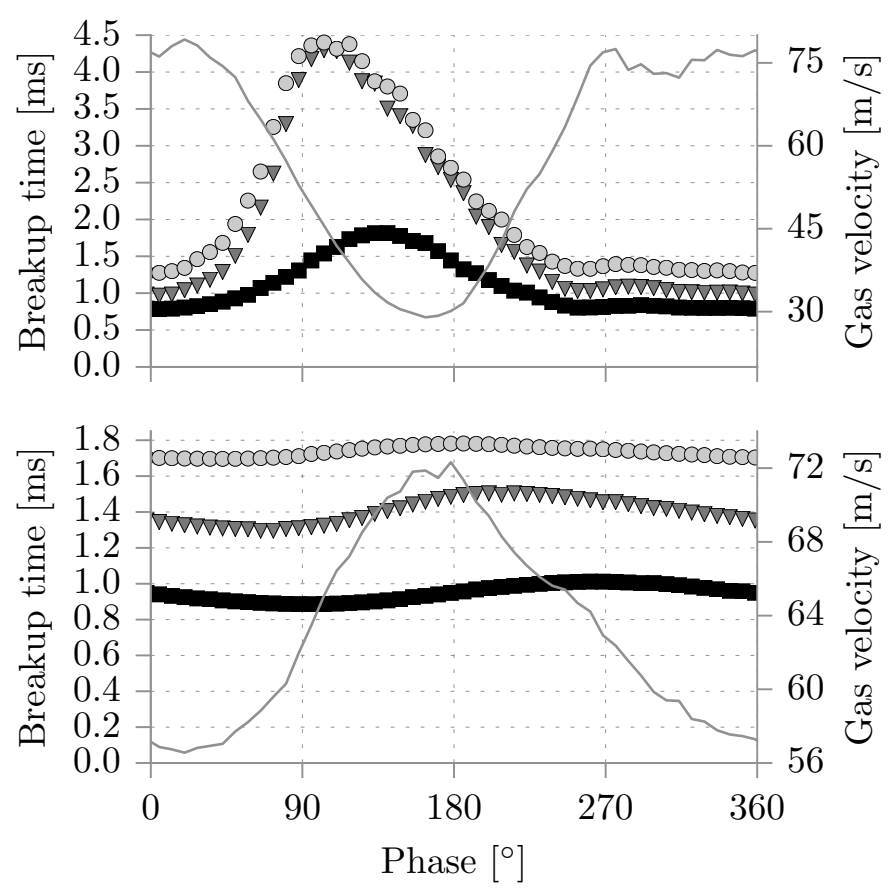

FIGURE 14: Breakup time including the velocity history predicted by Model $1(\mathbf{\square})$, Model $2(\nabla)$ and Model $3(\bigcirc)$ superimposed with the gas velocity (line), at $f=62 \mathrm{~Hz}$ (top) and $f=500 \mathrm{~Hz}$ (bottom).

Figure 15 displays the SMD computed with the averaging procedure for $f=62 \mathrm{~Hz}$ (top) and $500 \mathrm{~Hz}$ (bottom). At the low frequency, the averaging procedure improves considerably the results, especially for Model 2 and 3 which show a time response that is in good agreement with the experiment. On the contrary, Model 1 displays a discrepancy to the experiment larger than without the averaging procedure, with a SMD peaking at $\varphi=180^{\circ}$ compared to $210^{\circ}$ in the experiment. At high frequency, the improvement of the method is visible for Model 1 and 2, which show a time response and a signal amplitude comparable to that of the experiment. Model 3 shows a phase shift of $\approx 90^{\circ}$ and a lower amplitude, suggesting a too long averaging period, i.e. an overestimated breakup time. Finally, even though the SMD prediction of Model 1 shows a large deviation in steady state (Fig. 8), it shows significantly better results in transient regime.

Based on the only observation of the phase shift, Model 2 provides the breakup time that is in best agreement with the experi- ment, without any further fitting. This suggests that the breakup mechanism provided by Eckel et al. [16] might be closer to reality, even though it shows large deviation of the SMD at high frequency.
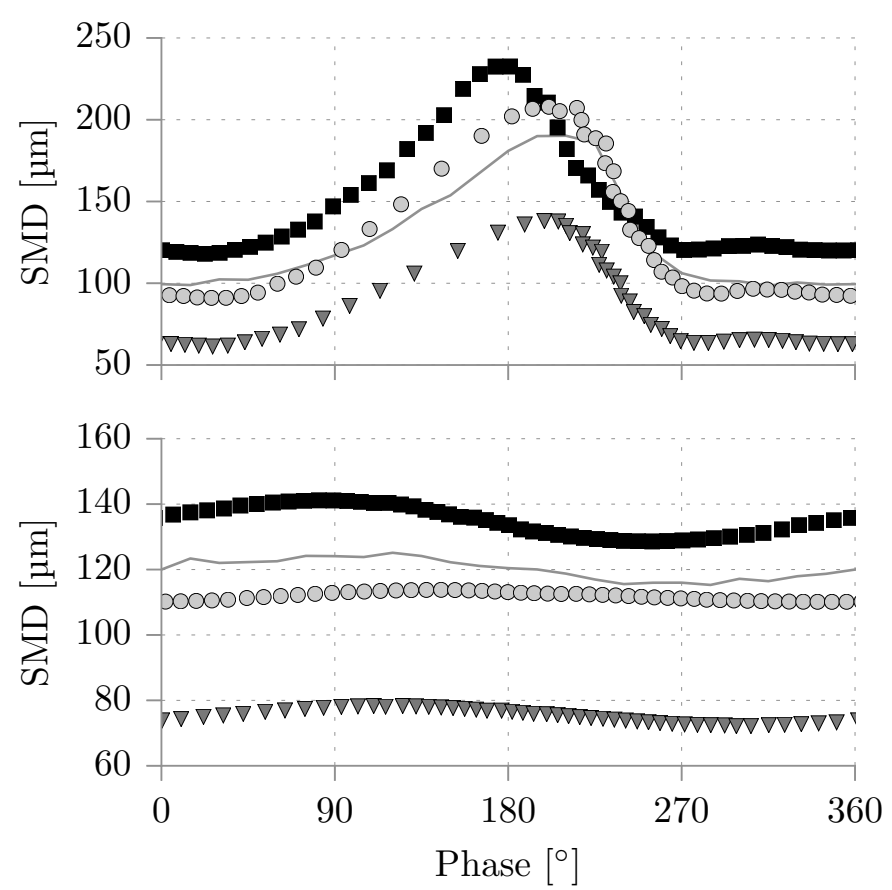

FIGURE 15: SMD including the velocity history predicted by Model $1(\mathbb{\square})$, Model $2(\nabla)$ and Model $3(\odot)$ and measured in the experiment (line) at $f=62 \mathrm{~Hz}$ (top) and $f=500 \mathrm{~Hz}$ (bottom).

\section{CALIBRATION OF MODEL TIME CONSTANTS}

The time constants of Models 1 to 3 are $\eta_{0}$ (Eq. 2), $C_{3}$ (Eq. 5a) and $C_{B U}$ (Eq. 9), respectively. They were calibrated, independently from the other models constants (referred to as steady state constants in the following), to match the experimental SMD for $f=62$ and $500 \mathrm{~Hz}$ with two different methods. First, the calibration was made with the original steady state constants, depicted for instance in Fig. 8. Second, the calibration was made with steady state constants calibrated on Müller's experiment in static conditions, depicted in Fig. 10. In the second calibration method, it appeared that Model 1 poorly matched the experiment, Model 2 was improved, and Model 3 was not significantly better. In order to depict the capability of the three models, the results of Model 1 and Model 3 will be only discussed with the time constant calibration (first method) and the results of Model 2 will be discussed with the full calibration (second method). The calibrated time constants were 10.9, 5.05 and 8.97 for Models 1 to 
3 , respectively.

The SMD predicted by the three models is plotted versus the phase angle for four fluctuation frequencies $f=62,125,250$ and $500 \mathrm{~Hz}$ in Fig. 16. At $f=62$ and $125 \mathrm{~Hz}$, which corresponds to the range of the rumble phenomenon, the global agreement is very good in terms of SMD and phase angle. It demonstrates the ability of the three models to render a correct SMD transient atomization. At $f=250 \mathrm{~Hz}$, Model 1 shows a very good agreement of the SMD variation with the phase angle, and an absolute SMD prediction shifted by $\approx+20 \mu \mathrm{m}$. On the contrary, Model 2 and Mode 3 show a correct absolute SMD prediction and a constant phase shift of $\approx+40 \mathrm{~Hz}$. The same behavior for Model 1 is observed at $f=500 \mathrm{~Hz}$ with a correct in-phase SMD variation and constant shift of the diameter by $\approx+20 \mu \mathrm{m}$. Although Model 2 and Model 3 show a lower absolute deviation of their predicted SMD, they show constant shifts both in phase $\left(\approx 90^{\circ}\right)$ and SMD $(\approx-5 \mu \mathrm{m})$. Despite these small deviations, the global agreement of the three models depicted in Fig. 16 proofs that considering prefilming airblast atomization as a low-pass filter is an appropriate assumption. Hence, the appropriate temporal mean of the gas velocity is sufficient to predict a correct SMD in transient state atomization.

\section{CONCLUSION}

The VPDF of a spray generated by a planar prefilming airblast atomizer in an oscillating flow was measured by the means of shadowgraphy. The velocity fluctuations frequency was varied between 62 and $500 \mathrm{~Hz}$, and the air velocity was measured by LDA. It appeared that at high gas fluctuation frequencies, the SMD of the spray exhibit almost no fluctuations. This observation allows to consider the accumulation breakup as a non-linear transfer function $F(t)=S M D(t) / u_{g}(t)$ with a low-pass behavior. It was also showed that up to $f=250 \mathrm{~Hz}$, a part of the continuous atomization process can be described with a steady state approach whereas at $f=500 \mathrm{~Hz}$ a completely unsteady atomization is observed.

Three recent models that aim at predicting the VPDF of a spray generated by a prefilming airblast atomizer were presented. In the context of implementing theses models into a CFD code, it was shown that Model 2 needs an extra fitting loop for each breakup event to match the SMD of the distribution to predicted SMD, which significantly increases computational overheads. Only the Model 3 proposes a local formulation for gas velocity. These models were tested with a constant as well as a fluctuating gas velocity. In the case of constant velocity, Model 2 and Model 3 showed a good agreement with the experiment without further calibration, most presumably because they were developed based on a very similar experiment. Model 1 globally over-predicted mean diameters of the spray, despite the use of a secondary breakup model (TAB) that effectively downsizes the spray droplets. A calibration of model constants within the ob-
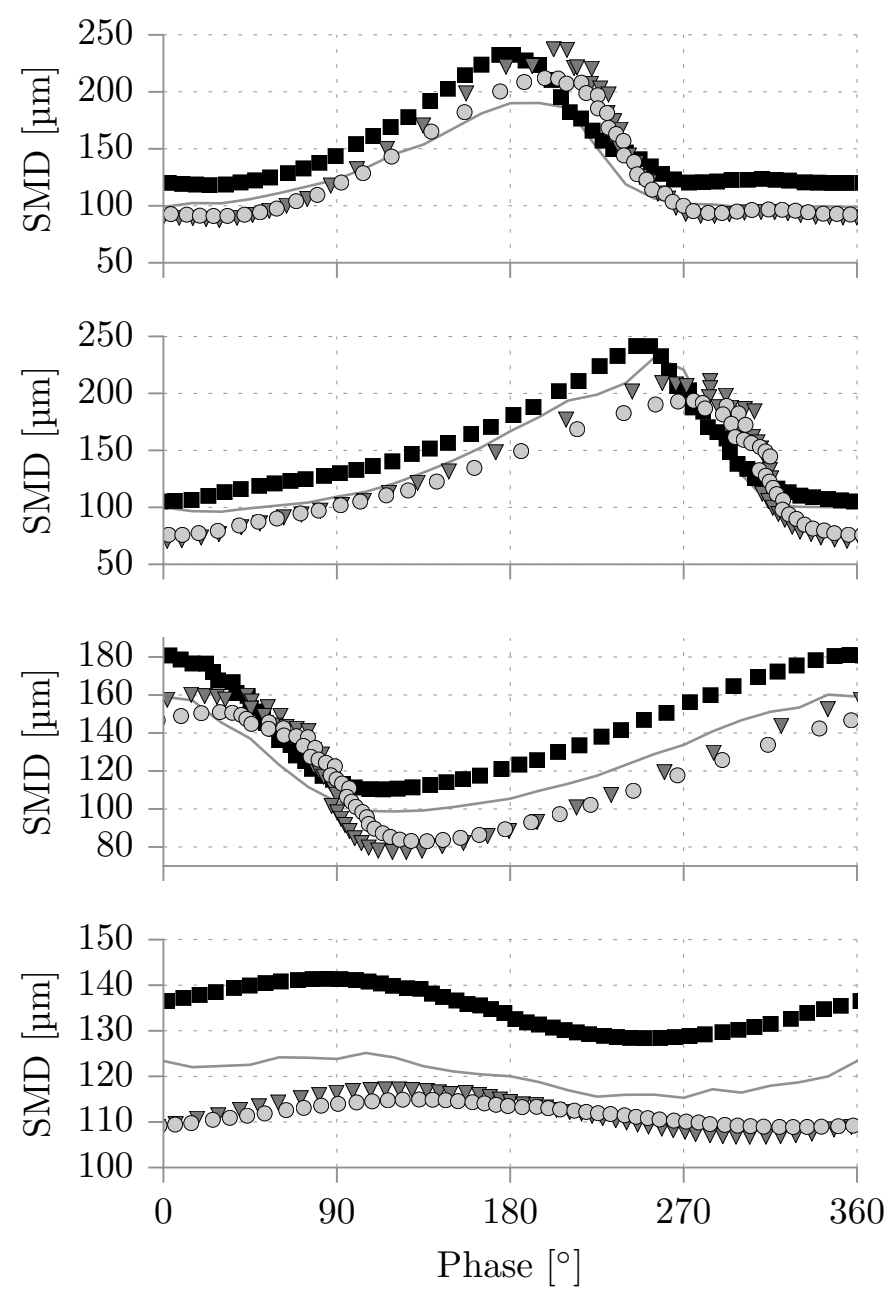

FIGURE 16: SMD predicted by Model $1(\mathbf{\square})$, Model $2(\boldsymbol{\nabla})$ and Model $3(0)$ with calibrated time constant, superimposed with the experiment (line) at $f=62,125,250$ and $500 \mathrm{~Hz}$ from top to bottom.

jectives to retrieve the proper experimental VPDF showed that the formulation of Model 1 failed to obtain a satisfactory agreement.

The application of these models to a fluctuating gas velocity revealed that using the instantaneous velocity leads to a strong discrepancy with the experiment, especially at high frequency. It was shown that taking the history of velocity into account via time-integration allowed to retrieve a better agreement with the experiment for all frequencies and for the three models. Finally it was shown that Model 1 and Model 3 only needed a calibration of their time constant to provide a correct estimation of the SMD in transient atomization, while Model 2 needs a steady state calibration prior to the fitting of its time constant. The global good agreement of the three calibrated models for the broad range of 
frequencies shows the validity of integrating the gas velocity to predict a correct SMD in transient atomization.

\section{ACKNOWLEDGMENT}

The authors like to thank the Helmholtz Association of German Research Centres (HGF) for funding. The authors also thank the anonymous reviewer for the suggestion of Fig. 4.

\section{References}

[1] Dowling, A. P., and Stow, S. R., 2003. "Acoustic analysis of gas turbine combustors". Journal of propulsion and power, 19(5), pp. 751-764.

[2] Eckstein, J., Freitag, E., Hirsch, C., Sattelmayer, T., Von der Bank, R., and Schilling, T., 2003. "Forced low-frequency spray characteristics of a generic airblast swirl diffusion burner". In Proceedings of GT2003, ASME Turbo Expo 2003: Power for Land, Sea and Air, pp. 471-478.

[3] Greenhalgh, D. A., Jermy, M. C., Doherty, W. G., and Hussain, M., 2004. "The response of fuel injector sprays to acoustic forcing". In ASME 7th Biennial Conference on Engineering Systems Design and Analysis, pp. 513-520.

[4] Müller, A., Koch, R., H.-J., B., Hehle, M., and Schäfer, O., 2006. "Performance of prefilming airblast atomizers in unsteady flow conditions". In Proceedings of GT2006, ASME Turbo Expo 2006: Power for Land, Sea and Air.

[5] Gajan, P., Strzelecki, A., Platet, B., Lecourt, R., and Giuliani, F., 2007. "Investigation of spray behavior downstream of an aeroengine injector with acoustic excitation". Journal of propulsion and power, 23(2), pp. 390-397.

[6] Bärow, E., Koch, R., and Bauer, H., 2013. "Comparison of oscillation modes of spray and gaseous flames". In Eighth Mediterranean Combustion Symposium.

[7] Gepperth, S., Guildenbecher, D., Koch, R., and Bauer, H.J., 2010. "Pre-filming primary atomization: Experiments and modeling”. In International Conference for Liquid Atomization and Spray Systems, ILASS-Europe.

[8] Gepperth, S., Müller, A., Koch, R., and Bauer, H.-J., 2012. "Ligament and droplet characteristics in prefilming airblast atomization". In ICLASS, 12th Triennial International Annual Conference on Liquid Atomization and Spray Systems.

[9] Müller, A., 2015. "Experimentelle Untersuchung des Zerstäubungsverhaltens luftgestützter Brennstoffdüsen bei oszillierenden Strömungen”. PhD thesis, Karlsruhe Institute of Technology.

[10] Babinsky, E., and Sojka, P., 2002. "Modeling drop size distributions". Progress in Energy and Combustion Science, 28, pp. 303-329.

[11] Inamura, T., Shirota, M., Tsushima, M., Kato, M., Hamajima, S., and Sato, A., 2012. "Spray characteristics of pre- filming type of airblast atomizer". In ICLASS, 12th Triennial International Annual Conference on Liquid Atomization and Spray Systems.

[12] Marmottant, P., and Villermaux, E., 2004. "On spray formation”. Journal of Fluid Mechanics, 498, pp. 73-111.

[13] O’Rourke, P. J., and Amsden, A. A., 1987, Paper 872089. "The TAB method for numerical simulations of spray droplet breakup". Society of automotive engineers, 96.

[14] Boukra, M., Cartellier, A., Ducasse, É., Gajan, P., Lalo, M., Noel, T., and Strzelecki, A., 2009. "Use of Faraday instabilities to enhance fuel pulverisation in air-blast atomisers". Comptes Rendus Mécanique, 337(6), pp. 492-503.

[15] Weber, C., 1931. "Zum Zerfall eines Flüssigkeitsstrahles". Zeitschrift für angewandte Mathematik und Mechanik, 11 (2), pp. $136-154$.

[16] Eckel, G., Rachner, M., Le Clercq, P., and Aigner, M., 2013. "Semi-empirical primary atomization models for transient Lagrangian spray simulation". In 8th International Conference on Multiphase Flow.

[17] Tate, R., and Marshall, W., 1953. "Atomization by centrifugal pressure nozzles". Chemical Engineering Progress, 49(4), pp. 169-174.

[18] Hsiang, L. P., and Faeth, G. M., 1992. "Near-limit drop deformation and secondary breakup". International Journal of Multiphase Flow, 18(5), pp. 635-652.

[19] Chou, W.-H., and Faeth, G., 1998. "Temporal properties of secondary drop breakup in the bag breakup regime". International Journal of Multiphase Flow, 24(6), pp. 889-912.

[20] Wert, K., 1995. "A rationally-based correlation of mean fragment size for drop secondary breakup". International Journal of Multiphase Flow, 21(6), pp. 1063-1071.

[21] Chaussonnet, G., Riber, E., Vermorel, O., Cuenot, B., Gepperth, S., and Koch, R., 2013. "Large Eddy Simulation of a prefilming airblast atomizer". International Conference on Liquid Atomization and Spray Systems.

[22] Chaussonnet, G., Vermorel, O., Riber, E., and Cuenot, B., 2016. "A new phenomenological model to predict drop size distribution in large-eddy simulations of airblast atomizers". International Journal of Multiphase Flow, 80, pp. 29-42.

[23] Holz, S., Chaussonnet, G., Gepperth, S., Koch, R., and Bauer, H.-J., 2016. "Comparison of the primary atomization model PAMELA with drop size distributions of an industrial prefilming airblast nozzle". In International Conference for Liquid Atomization and Spray Systems, ILASS-Europe. 